generic, and that what is involved in (1) is an inductive function $\zeta$ of $\xi$, viz.

$$
\zeta(n)=\xi(n) \xi(n-1) \cdots \xi(1) \lambda=\xi(n) \cdot \zeta(n-1) ;
$$

hence $\zeta$ is a generic and therefore stochastic sequence. This remarkable proof sheds new light on the theorem by showing that what makes it work is the inductive character of the underlying function.

\title{
IV. Concluding Remarks
}

The limitations of the author's prediction theory for individual time-sequences should be evident. Finite-valued sub-Markoff sequences are of no particular interest for prediction, and so the author's final theorem ( $\$ \mathrm{~F}$, above) is not very exciting. It is not yet clear if further work with the author's concept of "statistical prediction" will yield anything worthwhile in the actual analysis of the timesequences which arise in science. As these limitations could not possibly have been foreseen, the author has of course rendered yeoman service to the subject by venturing on this new frontier.

The wider concept of prediction advocated by the author has already proved fruitful in revealing the essential core of certain deep theorems in analysis and probability. But it remains to be seen if significant tracts of mathematical territory can be illuminated by the development of his ideas and techniques. The reviewer would certainly hope that this may be possible. Recently it has become clear that many linear prediction problems are special (deficiency 1) cases of other quite important problems in functional analysis, and that linear prediction techniques extend to the latter. It would be very satisfying indeed, if the same situation were found to prevail at the non-linear level.

Reading the book is not an easy job. The subject is itself rather hard. But part of the difficulty stems from the peculiar organization of the book, which is very different from that of this review, for instance. Idiosyncrasies in its format also make for hard reading, especially the absence of an index and misuse of the decimal system of enumeration. (Theorem 10.2 occurs in $\$ 10.4$ !) But the work stands as a first-rate and highly original dissertation on a very difficult subject.

P. MASANI

Strukturtheorie der Wahrscheinlichkeitsfelder und -räume. By D. A. Kappos. Ergebnisse der Mathematik, und Grenzgebiete, Heft 24. Springer, Berlin, 1960. 4+136 pp. DM 21.80 .

This book is the first to be devoted to a systematic account of the applications of Boolean algebras to measure theory. The direct con- 
nection to probability theory suggested by the title is limited to (i) restricting the measures considered to be of total weight one, (ii) a detailed treatment of the dual notions of Cartesian products and of independence, and (iii) a brief final chapter on conditional probability. Nevertheless, probability theorists should not be discouraged from reading the book, as it contains a valuable and self-contained treatment of an aspect of measure theory which is highly relevant to the foundations of their subject. Moreover, Professor Kappos has done an excellent job of assembling interesting and unfamiliar material in his book. His principal topic is one which had previously been treated only indirectly in the literature, namely, the relation between Boolean algebras and their subalgebras, especially when the algebras carry a measure. Most of the material covered has not appeared in book form at all, and much has appeared, even in the form of research papers, only within the last ten years. In addition, the style is lucid, and there are many illuminating examples. While intended primarily for specialists, the book can be read with profit by non-specialists as well. In particular, no previous familiarity with Boolean algebras is required; there is an adequate review of their properties in an appendix, which some readers may prefer to read first. Of course, considerations of space mean that many important topics had to be excluded. However, it is perhaps unfortunate that many excluded topics are not even hinted at, and that Professor Kappos has not always taken the opportunity to point out unsolved problems. This, however, is a minor objection to an excellent book.

A brief résumé of the contents follows: In Chapter I, $W$-fields or probability fields (i.e. finitely additive reduced measure algebras of total weight one) and their basic properties are established; included here is the representation of Boolean algebras by fields of sets. Countable operations and $\sigma-W$-fields are introduced in Chapter II. It is shown (by using the usual metric on the $W$-field) that every $W$-field can be imbedded in a $\sigma$-W-field, and criteria are given for a Boolean algebra to admit a $\sigma$-W-measure. In Chapter III, $W$-spaces are introduced; the chief concern here is with the extension of a finitely additive $W$-measure to a countably additive one. It is proved, via the Loomis representation theorem, that every $\sigma$-W-field arises from a $\sigma-W$-space. Chapters IV, V, and VI are concerned with the formation of product fields and spaces, various notions of independence of collections of subfields of a given $W$-field, and with extensions of probability measures from a given $W$-field to a containing field. In Chapter VI, there is also an account of the Kakutani-Oxtoby construction of an invariant inseparable extension of the Lebesgue meas- 
ure on the line. Chapter VII is devoted to the work of the Polish school on compact and quasi-compact measures, and Chapter VIII to conditional probabilities.

Dorothy MAHARAM

Stability by Liapunov's direct method with applications. By Joseph La Salle and Solomon Lefschetz. Academic Press, New York, 1961. vii +133 pp.

The stability criteria of Lyapunov's Second or Direct Method have been largely unknown to engineers in this country until very recently. The fact that they begin to be used now is in no small measure due to the authors' persistent efforts to acquaint engineers and mathematicians alike with this area of stability theory, which was developed almost entirely in the Soviet Union. The present monograph, the first on this subject written in English, is an outgrowth of these efforts. It is frankly aimed at the engineer with modest mathematical background. Thus, the principal results of Lyapunov's stability theory are presented with a minimum of technical detail; proofs of theorems when given are formulated in geometric rather than analytic language; examples selected with a view towards applications are completely worked out in the text. The pace is leisurely throughout, the exposition uncluttered and easily readable.

Chapter 1, entitled "Geometric concepts: Vectors and matrices," contains introductory material on vectors, matrices, quadratic forms and Euclidean geometry needed in the sequel. Chapter 2 on "Differential equations" forms the core of the book. Here the authors introduce the concepts of stability for a solution of an autonomous vector equation, define Lyapunov functions for such equations and prove the classical theorems of Lyapunov and Cetaev. The corresponding theorems for nonautonomous equations are mentioned only briefly. The construction of Lyapunov functions is illustrated in a number of examples of equations of second order and systems of first order, including the so-called critical case. A thorough discussion of the regions of stability, here called the extent of stability, follows. It includes proofs of the important generalizations of Lyapunov's theorems on asymptotic stability which do not require negative-definiteness of the time derivative. Chapter 3, entitled "Application of Liapunov's theory to controls," takes up a detailed study of linear control systems with continuous servo characteristic. Explicit stability criteria are derived from a suitable Lyapunov function whose construction is carried out in several cases. In Chapter 4, concerned with "Extensions of Liapunov's method," the authors discuss the use of differ- 